\title{
Impronta genética institucional y desarrollo de identidad internacional: estudio bibliométrico sobre médula- espinal-gato en Cinvestav
}

\author{
Francisco Collazo Reyes \\ María Elena Luna Morales \\ Evelia Luna Morales *
}

Artículo recibido:

13 de junio de 2014.

Artículo aceptado:

2 de diciembre de 2014.

\section{Resumen}

Se estudió la literatura generada en aspectos neurofisiológicos de la médula espinal y el gato como modelo experimental. Una práctica de investigación temprana como producto de la impronta genética institucional. El objetivo es analizar el cuerpo de literatura como un evento de comunicación científica, para mostrar cómo cada publicación arbitrada aporta al desarrollo de un sentido de identidad internacional del trabajo del Departamento de Fisiología y del Cinvestav. Se analizó la conformación de relaciones y estructuras de comunicación científica a través de redes sociotécnicas quinquenales así como el peso de la aportación institucional a la literatura global en el tema. La asociación del tema de la médula espinal, el gato como modelo experimental y P. Rudomín como autor más productivo se han mantenido vigentes en la literatura científica del

* Los tres autores pertenecen al Cinvestav, México. (fcollazo@fis.cinvestav.mx); (meluna@cinvestav.mx); (eluna@cinvestav.mx)

INVESTIGACIÓN BIBLIOTECOLÓGICA, Vol.30, Núm.70, septiembre/diciembre, 2016, México, ISSN: 0187-358X. pp. 41-60 
tema por más de 50 años. Durante este periodo, esta asociación ha desarrollado patrones de afiliación institucional al sistema de comunicación científica internacional, dando lugar al desarrollo de una de las líneas de investigación más simbólicas de la impronta genética en el Cinvestav.

Palabras clave: Estudio bibliométrico; Indicadores bibliométricos; Médula espinal; Modelos experimentales; Gato; Cinvestav-Departamento de Fisiología; Pablo Rudomín; Redes-Sociotécnicas.

\section{Abstract}

Institutional genetic imprinting and development of international identity: bibliometric study on spinalcord-cat in Cinvestav

Francisco Collazo-Reyes, María-Elena Luna-Morales and Evelia Luna-Morales

A corpus of literature addressing the neurophysiology of the spinal cord and the cat as an experimental mod$\mathrm{el}$ are examined. An early research practice as product of genetic institutional imprinting. The objective is to analyze the body of literature as a scientific communication event, showing how each peer reviewed paper contributes to developing a sense of international identity in the Department of Physiology and Cinvestav. The formation of relationships and structures of scientific communication through quinquennial socio-technical networks and the weight of institutional contribution to the global literature on the subject are analyzed. The thematic association of the spinal cord, the cat as experimental model and P. Rudomín, as the most productive author, have remained stable in indexes for over 50 years. During this period, the association has developed patterns of institutional affiliation with regard to international systems of scientific communication, giving rise to the development of one of the most symbolic lines of research in genetic imprinting in the Cinvestav.

Keywords: Bibliometric study; Bibliometric indicators; Spinal-Cord; Experimental models; Cat; Cinvestav-Department of Physiology; Pablo Rudomín; Sociotechnical-Networks. 


\section{INTRODUCCIÓN}

T a concepción del Centro de Investigación y de Estudios Avanzados del ـIPN (Cinvestav) lleva el espíritu naciente de la impronta genética de la ciencia de la posguerra. ${ }^{1}$ Un modelo institucional concebido para actuar en un periodo identificado como de transición de la pequeña a la gran ciencia $^{2}$ que surgió con un doble reto: construir prácticas científicas modernas que incidan, por un lado, en la solución de problemáticas locales y, por otro, generar resultados que aporten al acervo universal de conocimientos. ${ }^{3}$ Los principios y valores para afrontar estos retos están en la huella genética institucional, ${ }^{4}$ expresada en el decreto oficial de creación en el año de $1961^{5}$, así como en los patrones de comunicación científica, contenidos en una publicación temprana de A. Rosenblueth, J. Alanis y G. Pilar que marcó la fecha de ingreso del Cinvestav en los índices bibliográficos internacionales en el mismo año de su creación.

Por un lado, los valores explícitos en el decreto de creación incluyen criterios rigurosos de contratación de investigadores: formación a nivel doctorado, dedicación de tiempo completo, evaluación permanente y sin definitividad; libertad de cátedra y de investigación; participación en actividades de colaboración científica y la formación de nuevos investigadores; así como una administración basada en la aplicación de normas de gran sencillez. ${ }^{6}$ Por otro, las características del primer trabajo ${ }^{7}$ establecen seis estándares de prácticas internacionales de publicación: 1) difusión en revistas con arbitraje internacional; 2) colaboración internacional; 3) estructura de contenidos ajustada al formato científico IMRD: introducción, materiales, resultados y discusión; ${ }^{8} 4$ ) uso de referencias en fuentes acreditadas; 5) visibilidad internacional a través de citas, y 6) escritos en inglés. Estos aspectos corresponden a la emergencia en los modos de producir y certificar conocimientos en tiempos de la posguerra a nivel internacional. ${ }^{9} \mathrm{El}$ conjunto de relaciones sociales y científicas están

1 M. Albornoz, "La política científica y tecnológica en América Latina frente al desafío del pensamiento único".

2 D. J. de Solla Price, Little Science, big Science, y Little Science, big Science... and Beyond.

3 Manual de Santiago, Manual de Santiago de internacionalización de la ciencia y la tecnología.

4 Ibarrola, "La impronta genética del Cinvestav. Una mirada a la excelencia de la institución entonces y ahora"; B. R. Clark, The Higher Education System: Academic Organization in CrossNational Perspective.

5 Diario Oficial de la Federación, Decreto de la creación del CIEA, 5 noviembre de 1960.

6 Ibarrola, "La impronta genética...".

7 A. Rosenblueth, J. Alanis y G. Pilar, "The Accessory Motor Innervation of the Diaphragm”.

8 L. B. Sollaci y M. G. Pereira, "The introduction, methods, results, and discussion (IMRAD) structure: a fifty-year survey”.

9 R. Florida y M. Kenney, "Institutions and Economic Transformation. The Case of Postwar Japanese Capitalism”. 
orientadas a proveer el contexto institucional para el desarrollo científico, la formación de recursos y la continuidad de resultados. Estas características han identificado al Cinvestav con un modo diferenciado de hacer ciencia en América Latina y el Caribe. ${ }^{10}$ Los detalles de su evolución están en distintos esfuerzos dirigidos a constituir y vincular internacionalmente grupos o líneas de investigación, mismos que se han distinguido por mantener estabilidad en la generación de sus productos de investigación. Se trata de líneas de investigación como la médula espinal, que se han fortalecido en los procesos de afiliación a los estándares de las prácticas científicas internacionales. Generalmente, estos procesos siguen la vía del desarrollo de capacidades locales para acreditar resultados en el sistema internacional de conocimientos, a través de someter sus resultados al sistema de revisión de pares.

La concepción y materialización del Cinvestav coincidió en el tiempo con otros eventos importantes: a) la creación de los índices de citas desarrollados por el Institute for Scientific Information (ISI), que dieron origen al concepto de revistas de corriente principal de gran influencia en los mecanismos de acreditación de la ciencia; b) el incremento en el uso de grandes animales vertebrados como modelos de investigación, que influyeron en el crecimiento exponencial de la literatura en distintas áreas, entre ellas, la médula espinal. ${ }^{11}$ Las ventajas del gato como modelo experimental en el estudio de los aspectos neurofisiológicos de la médula espinal alcanzó su mejor momento, en términos de literatura científica registrada en los índices bibliográficos, en las primeras décadas de la segunda mitad del siglo XX. Distintas técnicas experimentales fueron desarrolladas en el gato antes de ser incorporadas a los modelos animales más pequeños. ${ }^{12}$ Una búsqueda en el sistema Scopus por los tópicos afferent, muscle, depolarization en combinación con spinal-cord y cat presenta una producción anual con una clara proyección de tipo exponencial durante el periodo 1960-1980, seguida de un largo periodo de producción decreciente hasta el año 2013 asociado a la pérdida de interés en el gato como modelo experimental y directamente relacionado con problemas de tipo social, económico, de mantenimiento, de tamaño del animal y de normatividad bioética, que han dificultado su uso en el laboratorio experimental.

El estudio de estos temas se ha abordado principalmente a través de métodos historiográficos, entre los cuales se encuentran los que utilizan como

10 H. Vessuri, "El Centro de Investigación y de Estudios Avanzados del IPN (Cinvestav)".

11 B. Rexed, "The Cytoarchitectonic Organization of the Spinal Cord in the Cat"; P. A. Guertin, "The Spinal Cord: Functional Organization, Diseases, and Dysfunctions".

12 A. Frigon, "The Cat Model of Spinal Cord Injury"; D. P. Cardinali, Neurociencia aplicada: sus fundamentos. 
unidad de análisis la literatura científica. El más utilizado está desarrollado con base en algoritmos y perfiles genealógicos de la evolución de los campos. ${ }^{13}$ Otros consideran distintos indicadores para analizar la evolución de los campos, orientados a identificar los cambios en los modos de conocer y realizar las prácticas científicas en cada disciplina. ${ }^{14}$ De acuerdo con otro método, como la teoría del "actor red", ${ }^{15}$ la dinámica de evolución de los campos es producto de muchas interacciones y elementos, explicados solamente por factores de orden social y técnico. ${ }^{16}$

En este trabajo se utilizan los artículos científicos como unidad de análisis. Nos apoyamos en el concepto de la intertextualidad referencial como una característica natural del género científico ${ }^{17}$ que nos ayuda a ponderar la fuerza operacional de los textos. ${ }^{18}$ De acuerdo con esta idea, cada nueva publicación aporta elementos a la construcción de redes de relaciones que se activan a través de las referencias, las citas y las coautorías. La continuidad en la publicación de resultados conforma tejidos de relaciones y flujos de comunicación que orientan los sentidos de la afiliación científica institucional. Según el tipo de revistas, pueden ser flujos que apuntan a discursos científicos contenidos en fuentes locales, regionales, internacionales o una combinación de éstos. De esta manera, las comunidades científicas construyen sus líneas de investigación, objetos de estudio y discurso en la medida que logran condiciones estables para mantener la continuidad en la producción de sus textos. ${ }^{19}$ Visto así, los textos se entienden como conocimientos socialmente construidos.

Se asume que la línea de investigación sobre la médula espinal asociada al gato como modelo experimental se ha significado a través de la repetición de marcas contenidas en los textos (el autor principal, las palabras más frecuentes, las revistas preferidas y el modelo) como un tema simbólico en el Departamento de Fisiología y el Cinvestav. Este sentido simbólico ha tenido

13 E. Garfield, A. I. Pudovkin y V. S. Istomin, "Why Do We Algorithmic Historiography"; L. Leydesdorff, "Eugene Garfield and Algorithms Historiography: Co-Words, Co-Authors and Journals”.

14 J. V. Pickstone, "Working knowledge before and after circa 1800: Practices and disciplines in the history of science, technology and medicine"; J. V. Pickstone, "A Brief Introduction to Ways of Knowing and Ways of Working".

15 M. Callon y B. Latour, "Unscrewing the big leviathan: How actors macrostructure reality and how sociologists help them to do so"; B. Latour, "On actor-network theory - A few clarifications".

16 J. Law, "Notes on the theory of the actor network-ordering, strategy, and heterogeneity".

17 A. J. Devitt, "Intertextuality in Tax Accounting; Generic, Referential and Functional".

18 S. Doheny-Farina, "Creating a text / Creating a Company. The role of a text in the Rise and Decline of a New Organization".

19 C. Bazerman, Shaping Writing Knowledge; C. Bazerman y J. Paradis, Textual Dynamics of the Professions. Historical and Contemporary Studies of Writing in Professional Communities; C. Berkenkotter, T. N. Huckin y J. Ackerman, "Social Context and Social Constructed Text". 
como escenario de desarrollo la formación de estructuras de comunicación científica internacional desarrolladas en la institución por más de 50 años.

En este trabajo utilizamos una metodología apoyada en tres aspectos complementarios: 1) una matriz de análisis que combina distintos elementos bibliográficos: autores, referencias, citas, coautoría, frecuencia de palabras y modelos experimentales utilizados; 2) el análisis de estos elementos organizados en 11 redes sociotécnicas quinquenales; 3) el estudio de la producción científica internacional para determinar las posiciones que ocupan los autores, el Cinvestav y México en el tema médula-espinal-gato, de acuerdo con la cobertura del sistema Scopus. Este indicador de la aportación del Cinvestav y el Departamento de Fisiología al acervo universal de conocimientos en el área es también, de acuerdo con el Manual de Santiago, ${ }^{20}$ un indicador de internacionalización del quehacer institucional.

Encontramos que el estudio de los aspectos neurofisiológicos de la médula espinal, el gato como modelo experimental, el Cinvestav, el Departamento de Fisiología y P. Rudomín emergieron en la literatura científica al mismo tiempo y se han mantenido vigentes por un periodo mayor a 50 años (19612013). Durante este periodo la asociación del tema, el modelo experimental, los autores y la institución se han mantenido visibles en los índices bibliográficos y en los indicadores de productividad científica internacional. Esta asociación ha construido un tema de investigación en un escenario internacional y, a nivel local, una línea de investigación simbólica que le ha construido identidad internacional al Departamento de Fisiología y al Cinvestav.

\section{Metodología}

A partir de un trabajo previo sobre la producción del Departamento de Fisiología ${ }^{21}$ se identificó el primer grupo de investigación formado por A. Rosenblueth, J. García Ramos y P. Rudomín, que logró mantener continuidad en la publicación de resultados y dio origen a una línea de investigación que se ha mantenido vigente durante toda la historia del Cinvestav. Se utilizó como fuente de información la base de datos del Atlas de la Ciencia Mexicana, 1961-2013 (http://atlasdelacienciamexicana.org/). ${ }^{22}$ Se incluyeron los trabajos de los autores mencionados que utilizaron el gato como modelo experimental para el estudio de aspectos neurofisiológicos de la médula espinal.

20 Manual de Santiago, Manual de Santiago..., 111.

21 F. Collazo-Reyes, M. E. Luna-Morales y E. Luna-Morales, "Aproximación a las formas de organización de la producción científica a través de redes de coautoría”.

22 Atlas de la Ciencia Mexicana 2012: Bilingual edition. 
Se identificaron en total 132 trabajos a partir de los cuales se generaron dos archivos: el primero incluye las referencias hechas en ellos y el segundo las citas recibidas. Con estos archivos se desarrolló una base de datos local conformada con tres tablas relacionales: 1) trabajos publicados, 2) referencias incluidas, y 3 ) citas recibidas.

\section{Desarrollo de redes sociotécnicas por quinquenio}

A partir de la organización de los recursos de información se realizó un análisis histórico de la literatura. En este caso se dividió la producción científica por quinquenios y se utilizó como metodología de análisis las redes socio-técnicas. En consecuencia, se desarrollaron 11 redes, mismas que incluyen los siguientes componentes: 1) relaciones de coautoría de los trabajos publicados en el tema, 2) revistas de publicación, 3) número de referencias incluidas en los trabajos, 4) número de citas recibidas, 5) palabras más frecuentemente usadas en los títulos de los trabajos, y 6) modelos de experimentación. Las redes tienen la siguiente nomenclatura: los nodos de autores están representados por cuadrados, las revistas por rombos, el número de referencias por triángulos, el número de citas por círculos gris oscuro, las palabras por círculos gris claro y el modelo experimental por hexágonos. Todos los componentes de un mismo trabajo están relacionados por líneas, así como los autores o palabras que concurren en trabajos publicados en cada quinquenio. En cada periodo se analizaron las estructuras de relaciones de las coautorías; las revistas preferidas para publicar, para citar y las citantes; los promedios de referencias y citas por trabajo; la frecuencia de uso de las palabras y los modelos experimentales utilizados; así como la concurrencia de las palabras en dos o más trabajos. Lo anterior permitió caracterizar los patrones de comunicación que acompañaron la continuidad de la producción en la línea de investigación médula-espinal-gato en el Cinvestav.

Para el desarrollo de las matrices y las redes se utilizó el software Pajek (http://vlado.fmf.uni-lj.si/pub/networks/pajek/doc/pajekman.pdf), ${ }^{23}$ un programa desarrollado para el análisis de grandes redes.

\section{Aportación de la producción institucional a la ciencia internacional}

La internacionalización del trabajo de una institución de investigación tiene múltiples formas de expresión. Una de ellas es a través de la producción cien-

23 V. Batagel y A. Mrvar, Pajek. Program for Analysis and Visualization of Large Networks: Reference Manual. List of commands with short explanation version 2.05 . 
tífica de artículos publicados en revistas arbitradas. ${ }^{24}$ En este caso se utilizó el peso de la posición de los autores y la institución, asignadas en función de la producción científica en la clasificación internacional obtenida a través del sistema de información Scopus. De esta manera se determinó la posición de P. Rudomín y el Cinvestav en la literatura científica internacional. En este caso se realizaron búsquedas de información en el sistema Scopus, se utilizaron las palabras más significativas de los títulos como tópicos de información, combinadas con el tema spinal cord y el gato como modelo experimental, bajo tres distintas estrategias de búsqueda: 1) Afferent + spinal-cord and cat; 2) Muscle + spinal cord and cat; 3) Presynaptic and cat, y 4) Depolarization + spinal-cord and cat, todas en el periodo 1960-2013. En las tres búsquedas se refinaron los datos globales por países, instituciones y autores. El sistema Scopus organiza la información de acuerdo con la producción de los primeros 40 autores o instituciones más productivos. Se normalizó la información referente a los nombres de las instituciones de adscripción de los autores, y con base en la producción se determinaron las posiciones del Cinvestav y los autores.

\section{Resultados}

\section{Aspectos generales}

Se encontraron 132 trabajos que dan cuenta de la conformación de la línea de investigación sobre el tema médula-espinal-gato en el Cinvestav, construida a través del establecimiento de múltiples relaciones de colaboración, intertextualidad referencial y citación que involucra a los autores, las revistas y las palabras del discurso dominante en la literatura científica de corriente principal relacionada con el tema referido. Este tejido involucra miles de relaciones de influencia y de reconocimiento, establecidas a través de 4298 referencias hechas y de 3458 citas recibidas. Incluye 44 revistas preferidas para publicar, 444 citadas en las referencias y 360 que contienen los trabajos citantes. Existen cientos de autores involucrados en las referencias y en las citas. Estas relaciones de comunicación científica han mantenido visible al Cinvestav en los índices bibliográficos de la literatura científica de corriente principal durante más de 50 años. 


\section{Redes quinquenales}

La línea de investigación sobre médula-espinal tiene sus antecedentes en las prácticas de investigación realizadas en el Instituto Nacional de Cardiología. Se inició en el grupo de investigación formado bajo la influencia del prestigio internacional alcanzado por A. Rosenblueth. Las primeras redes presentan la formación de las estructuras de comunicación científica que caracterizan las prácticas internacionales de acreditación que siguieron los primeros trabajos de investigación generados en Cinvestav. La red del quinquenio 1961-1965 (Figura 1) muestra el grupo de investigación pionero en el tema. Incluye los primeros indicadores bibliométricos internacionales institucionales: 11 trabajos, escritos en coautorías por nueve autores principalmente: P. Rudomín, A. Rosenblueth y J. García Ramos; en cuatro revistas (una regional y tres internacionales); con 179 referencias que corresponden a trabajos publicados principalmente en las revistas Journal of Neurophysiology, Brain Research, Brain Research Bulletin, Archives Italiennes de Biologie, y Journal of Physiology; 117 citas recibidas hasta la fecha, hechas principalmente en Archives Italiennes de Biologie, Brain Research, Electro Encephalography and Clinical Neurophysiology, Acta Physiologica Latinoamericana, y Experimental Brain Research; un discurso caracterizado por seis palabras: afferent, arterial, blood, control, motor y pressure; y el gato como principal modelo experimental.

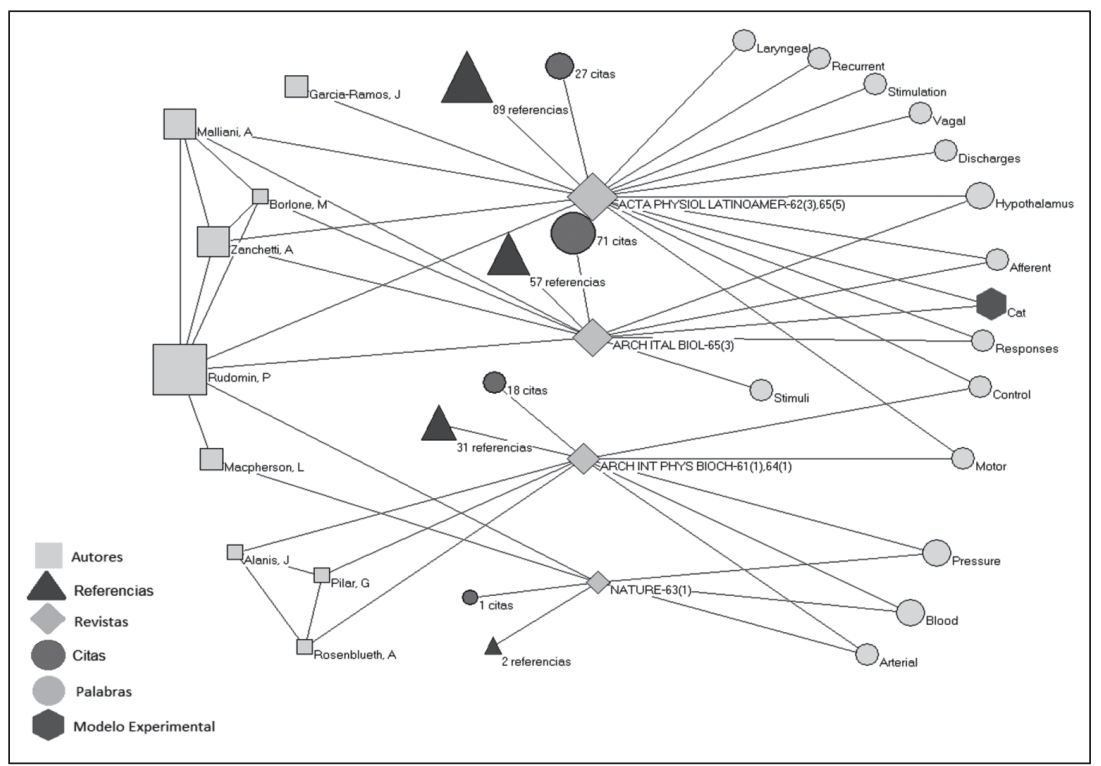

Figura 1. Red sociotécnica quinquenal: 1961-1965 
Las redes de los quinquenios 1966-1970, 1971-1975 y 1976-1980 presentan patrones de comunicación científica que mantienen las características del primer quinquenio. Se incrementó a 69 el número de trabajos y las citas a más de 1 000; una coautoría de 55 investigadores, 15 revistas y más de 1000 referencias. El discurso científico está centrado en las palabras afferent, depolarization, spinal, cord and cat.

Las cuatro primeras redes (1961-1980) comparten algunas características. Utilizan como medio principal de difusión la revista regional Acta Physiologica Latinoamericana; los autores más productivos y consistentes son $\mathrm{P}$. Rudomín y J. García Ramos; los trabajos más citados están publicados en Brain Research y las revistas citantes más importantes son Physiological Reviews, Journal of Neurophysiology y Brain Research. La continuidad en la publicación de la línea de investigación mantuvo visible al Cinvestav-Departamento de Fisiología en el Science Citation Index durante las décadas de los años 60 y 70.

A partir de la tercera red, 1971-1975, aparecen como coautores importantes S. Glusmann, R. Nuñez y J. Madrid, que son investigadores de las primeras generaciones de estudiantes formados en Cinvestav por P. Rudomín. La red 1976-1980 (Figura 2) registra el periodo más productivo asociado a la mayor diversificación de la procedencia de los autores. Coinciden los autores

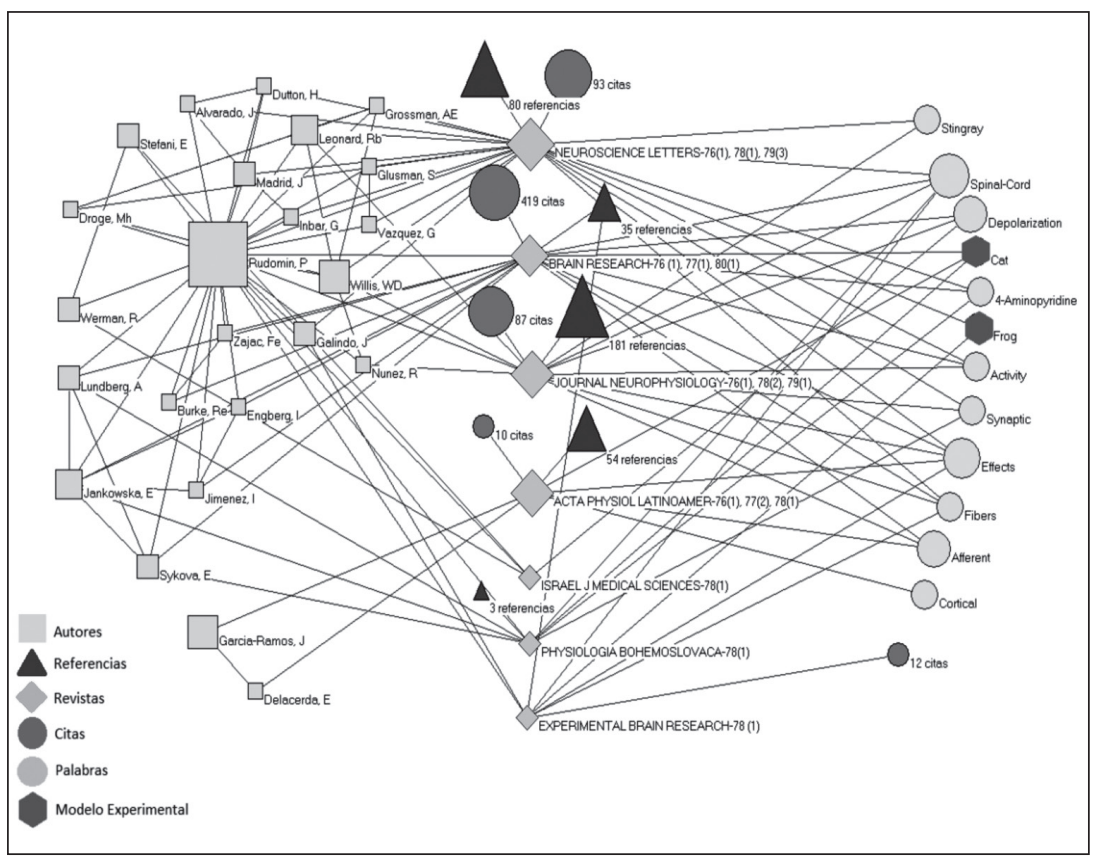


locales más tradicionales: P. Rudomín y J. García-Ramos, así como investigadores jóvenes formados en el Cinvestav (R. Nuñez, J. Madrid, S. Glusman, I. Jiménez, J. Galindo); además de autores adscritos a otros departamentos (H. Dutton) y autores de instituciones extranjeras ya reconocidos (F. E. Zajac) y principalmente coautores nuevos (R. Leonard, R. Werman, W. D. Willis, A. Lundberg, T. Jankowska, entre otros). El 79 \% de la producción está realizada en coautoría con P. Rudomín, quien se consolidó como el principal constructor de la línea de investigación. En este periodo aparece por primera vez la rana (frog) como modelo experimental.

Los patrones de comunicación de las redes de la década de los 80 presentan cambios en los patrones de comunicación (Figura 3). Dejó de aparecer la revista más productiva de las primeras dos décadas (Acta Pbysiologica Latinoamericana), así como su autor principal (J. García Ramos). La producción está basada principalmente en investigadores formados en Cinvestav. Las palabras spinal, cord, afferent, cat, depolarization, fibers, entre otras, conforman la matriz de palabras más utilizadas en los títulos, que identifican la literatura de este grupo de investigación en estos temas a nivel internacional. Los autores P. Rudomín, I. Jiménez y M. Solodkin se diferencian como la relación de colaboración más productiva. Incluye los trabajos más citados que alcanzaron promedios de citas y referencias por artículo, que se duplicaron en comparación con los indicadores obtenidos en los quinquenios anteriores.

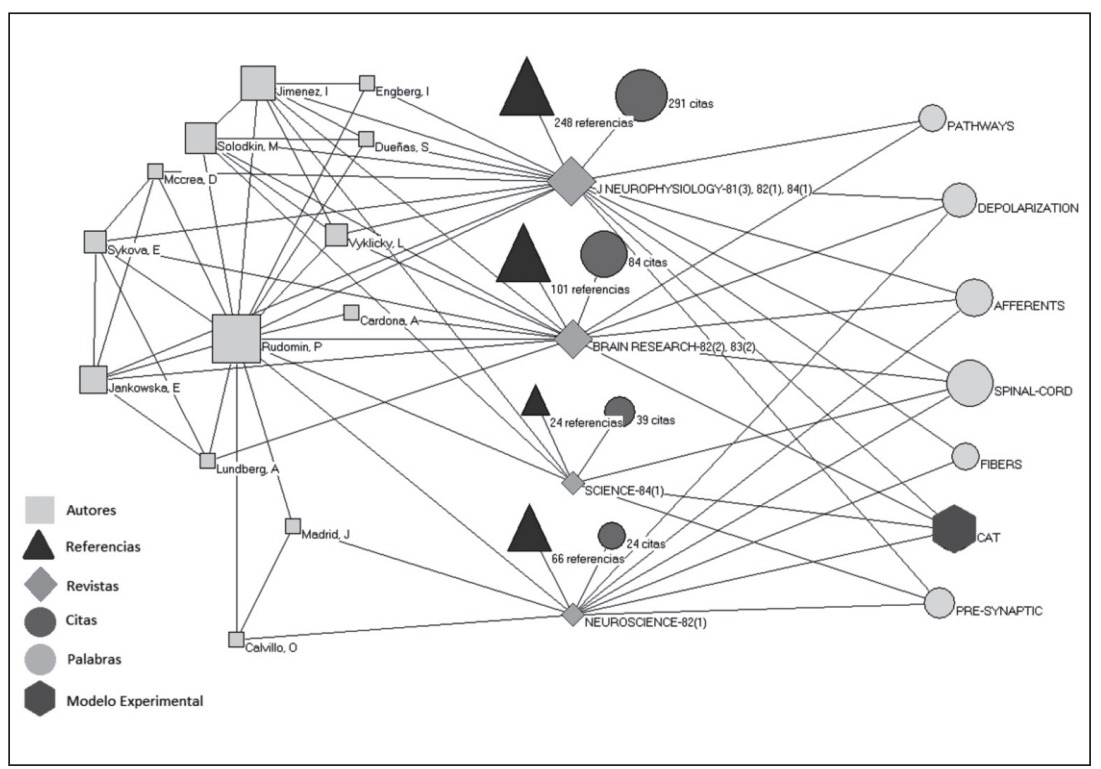

Figura 3. Red sociotécnica quinquenal: 1981-1985 
De acuerdo con la Figura 4, en la primera mitad de la década de los 90, con la misma base de autores formados en Cinvestav, se incrementó la producción y las revistas de difusión, pero se obtuvo uno de los promedios de citas más bajo. Cambiaron algunas palabras de la matriz tradicional, como stimulation, muscle, synaptic y presynaptic; otras como afferent, inhibition depolarization se mantuvieron vigentes.

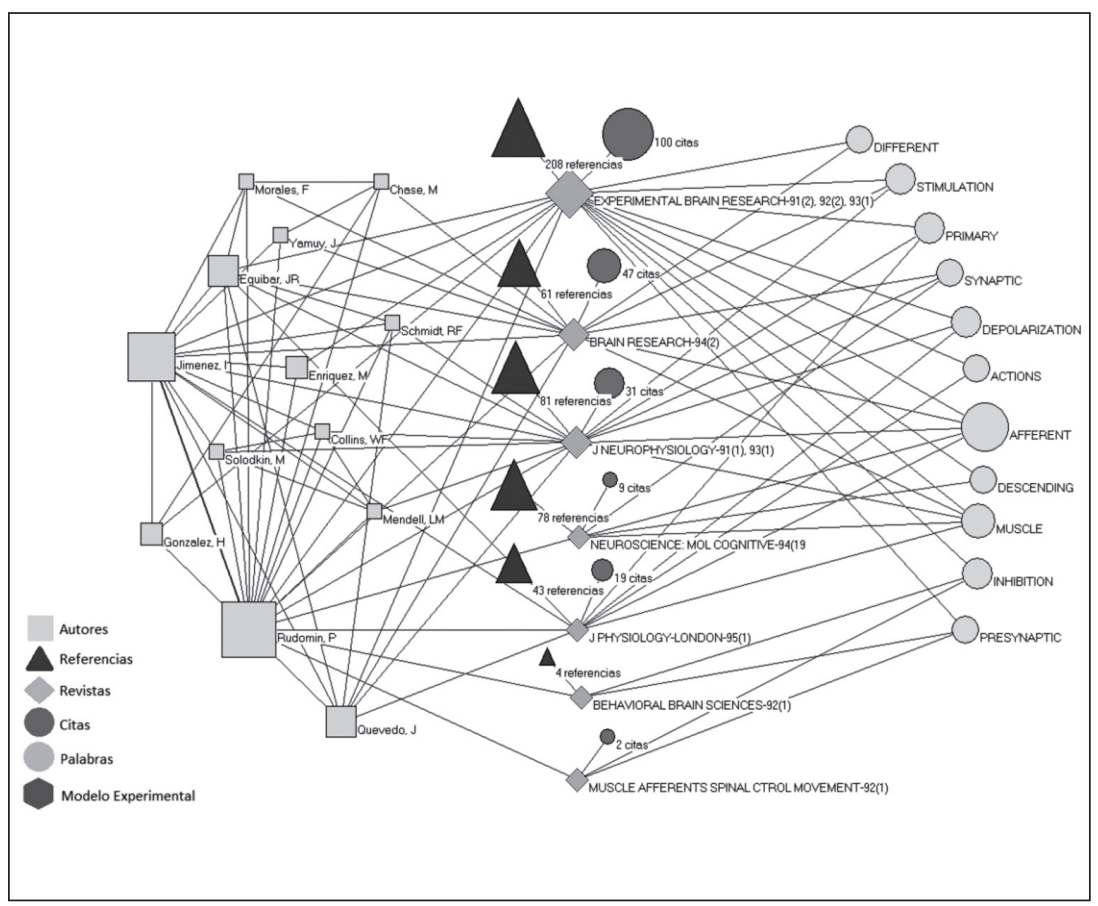

Figura 4. Red sociotécnica quinquenal: 1991-1995

Como se observa en las Figuras 5 y 6, con la producción del periodo 19962000 y 2001-2005 se retoma la matriz tradicional de palabras afferent, spinal, cord and cat, incluyendo los mismos patrones de comunicación de los quinquenios precedentes. Los trabajos más citados están publicados en Experimental Brain Research y realizados por investigadores formados en el Cinvestav. Estas redes mantienen los mismos núcleos de autores principales, revistas y promedios de referencias. En el periodo 2001-2005 disminuyó históricamente el promedio de citas por trabajo con respecto al quinquenio anterior. 


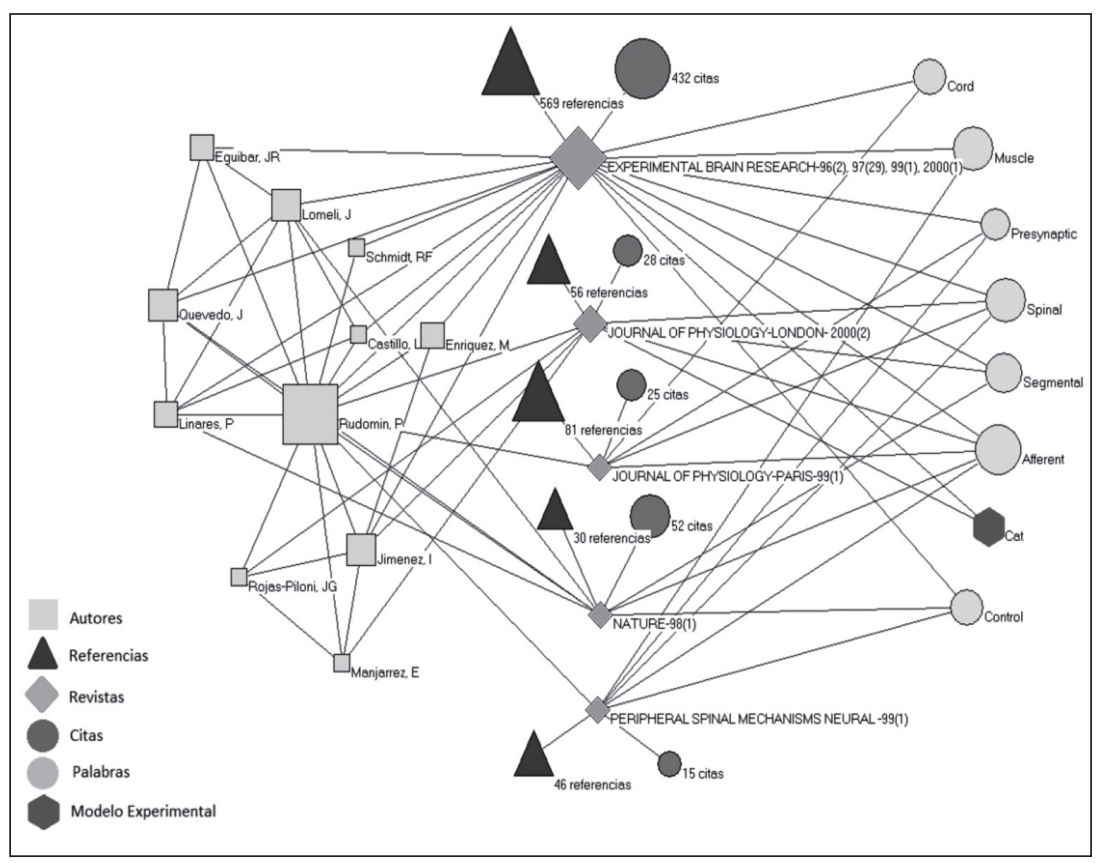

Figura 5. Red sociotécnica quinquenal: 1996-2000

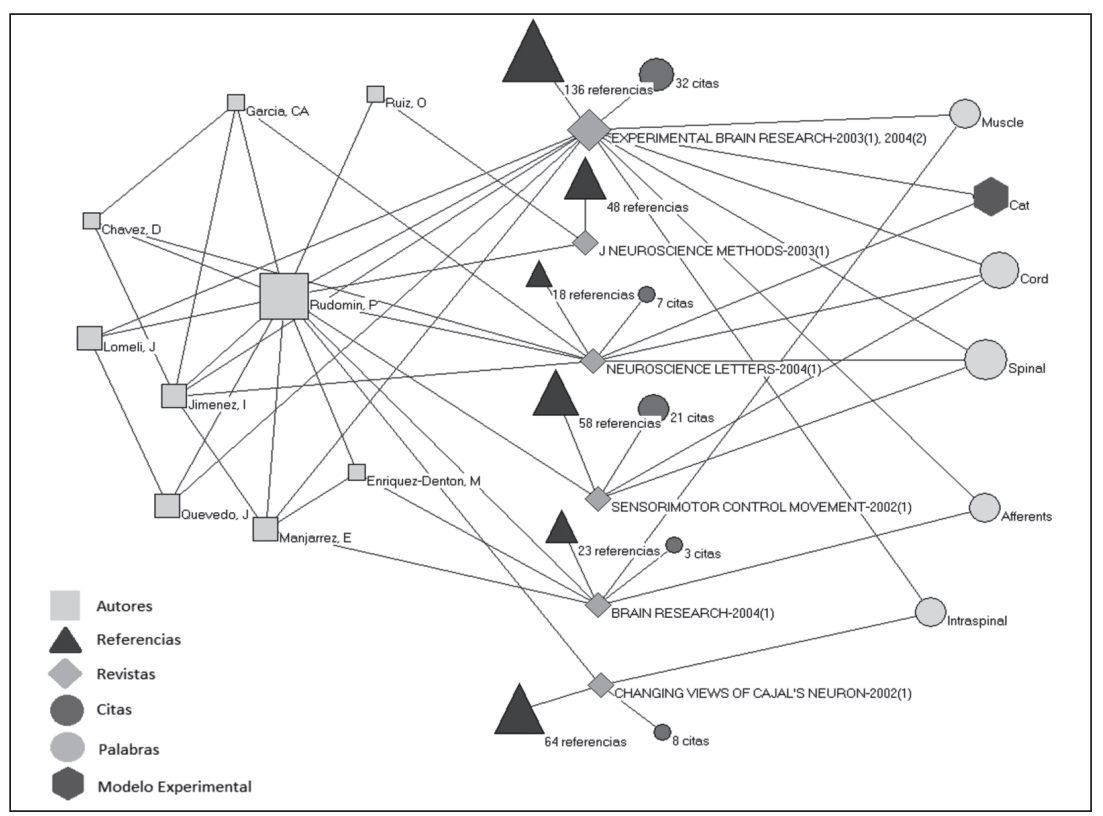

Figura 6. Red sociotécnica quinquenal: 2001-2005 
El quinquenio 2006-2010 presenta los patrones de comunicación más diferenciados en términos de producción (Figura 7). Cuatro trabajos, tres coautores, una revista (Experimental Brain Research) y tres palabras principales: afferent, joint and cat.

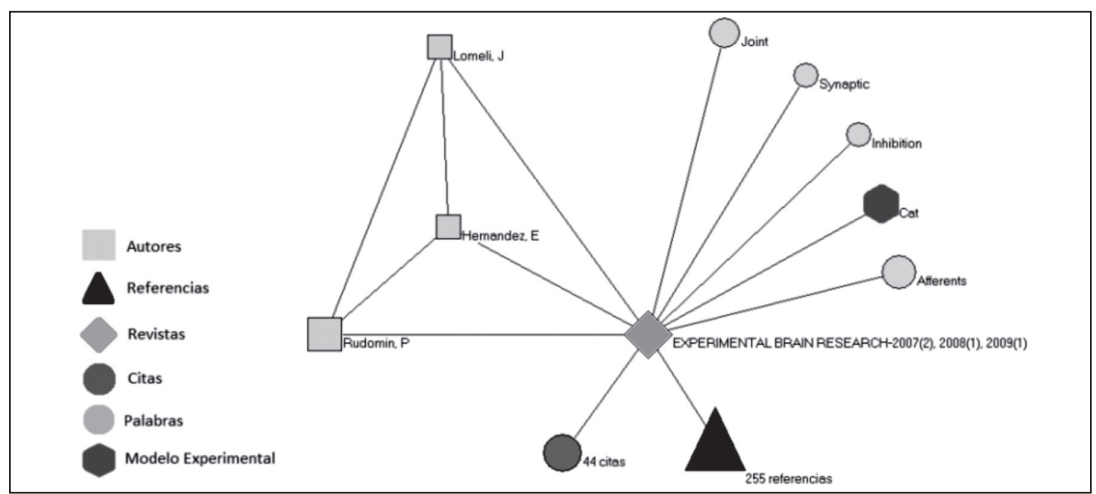

Figura 7. Red sociotécnica quinquenal: 2006-2010

La Figura 8 muestra la última red (2011-2013), donde predominan los autores centrales (P. Rudomín e I. Jiménez) y las palabras tradicionales del grupo de investigación, así como una de las revistas preferidas Journal Physiology. Aparecen también nuevos nombres de autores y revistas relacionadas con aspectos de ingeniería y computación, entre ellas una revista de acceso libre. Las características de las Figuras 7 y 8 se interpretan como señales de un cambio en los patrones tradicionales de comunicación.

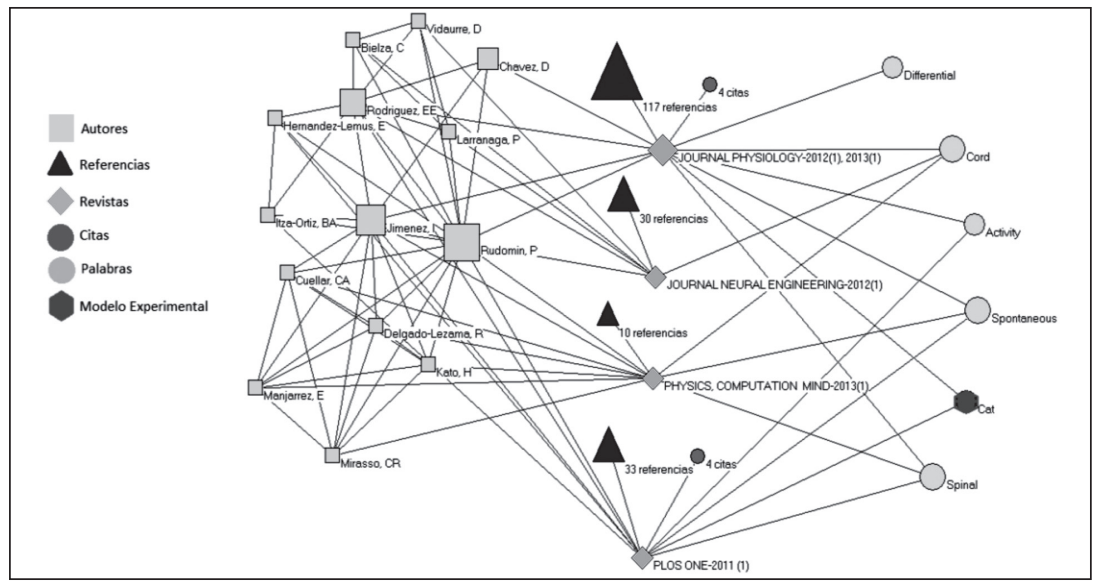


Las redes sociotécnicas de autores, revistas, citas, referencias y palabras muestran paso a paso los eventos de producción de sentido de la identidad internacional del Cinvestav. Las redes ofrecen información sobre aspectos que tienen que ver con distintas dimensiones de la internacionalización del trabajo institucional. En primer lugar, está el hecho de utilizar las revistas de mayor prestigio en el área: Journal of Neurophysiology, Experimental Brain Research, Journal of Physiology-London y Brain Research. Estos cuatro títulos se encuentran entre los cinco primeros lugares de las revistas, según las tres funciones que realizan en el sistema de comunicación: 1) como fuentes de publicación; 2) como fuentes citadas, y 3) como fuentes citantes. En segundo lugar, en la publicación de trabajos en coautoría participan 37 investigadores adscritos a instituciones extranjeras. En tercer lugar, se identificó un promedio alto de 26 citas por trabajo. Y en cuarto lugar, las revistas utilizadas para publicación están incluidas en los índices bibliográficos internacionales como revistas de corriente principal.

\section{Aportación institucional a la producción científica internacional}

La producción científica, como una de las expresiones de internacionalización institucional, se dimensionó a través de las posiciones de los autores y la institución en una clasificación de la producción internacional sobre el tema, obtenida del sistema Scopus. De acuerdo con la Tabla 1, la producción científica institucional generada en el tiempo ha posicionado internacionalmente a los autores, representados por P. Rudomín, y al Cinvestav entre los 10 primeros lugares de 40 en los tres tópicos analizados. En el tópico más representativo, Afferent + Spinal Cord + Cat, ocuparon los lugares dos y cuatro, respectivamente. Estas posiciones a nivel internacional tienen un peso específico, son indicativos de un nivel importante de aportación al acervo internacional de conocimientos en los tres tópicos.

Tabla 1. Indicadores de internacionalización de la producción científica sobre médula-espinal-gato, 1960-2013

\begin{tabular}{|l|c|c|c|c|c|c|}
\hline Núm. & \multicolumn{2}{|c|}{ Estrategia de búsqueda } & Producción & \multicolumn{3}{|c|}{$\begin{array}{c}\text { Posiciones en la literatura científica } \\
\text { internacional (Scopus) }{ }^{*}\end{array}$} \\
\hline & Palabras & Modelo & Palabras & \multicolumn{2}{|c|}{ Autor } & Cinvestav $^{\star *}$ \\
\hline 1 & $\begin{array}{c}\text { Afferent }+ \text { spinal } \\
\text { cord }\end{array}$ & Cat & 2,867 & P. Rudomín & 2 & 4 \\
\hline 3 & $\begin{array}{c}\text { Muscle }+ \text { spinal } \\
\text { cord }\end{array}$ & Cat & 2,864 & P. Rudomín & 6 & 10 \\
\hline & $\begin{array}{c}\text { Depolarization }+ \\
\text { spinal cord }\end{array}$ & Cat & 488 & P. Rudomín & 1 & 1 \\
\hline
\end{tabular}

Fuente: Scopus, 1960-2013.

* Scopus, incluye los primeros 40 lugares más representativos según producción científica.

** Producción científica registrada en Scopus. 


\section{Discusión}

La creación del Cinvestav, el inicio de los índices de citas, el incremento de la producción en el tema de la médula espinal y el uso del gato como modelo experimental, así como la adscripción al Cinvestav de un grupo de investigadores especializados en neurofisiología (A. Rosenblueth, J. García-Ramos y P. Rudomín) ${ }^{25}$ son eventos que coincidieron a principios de la década de los años 60 en la ciencia en México. Esta relación se ha mantenido vigente en los índices bibliográficos internacionales hasta el año 2013, un poco más de 50 años. Durante este periodo, la matriz de palabras afferent, médula y espinal; el gato como modelo experimental y el autor P. Rudomín se han significado en los indicadores de productividad científica del tema. El Cinvestav y el Departamento de Fisiología-México se han posicionado en el mapa de la geografía científica internacional como un importante nodo de flujo, atracción y producción de conocimientos en el tema.

Las redes sociotécnicas quinquenales dan cuenta del proceso de arraigo institucional de esta práctica de investigación. Está caracterizada por crear condiciones institucionales estables para mantener vigente la producción de conocimientos en temas neurofisiológicos de médula espinal durante toda la historia de la institución. En el escenario de la comunicación científica, este corpus de literatura está conformado por artículos, como unidades de comunicación, compuestos por distintos elementos: autores, revistas, citas, referencias, modelos experimentales y palabras. Lo que se puede advertir en las redes quinquenales es que cada vez que una nueva unidad de comunicación se publica influye en las estructuras de conformación de las redes: aparecen y desaparecen nodos y relaciones en las redes. Los componentes que se repiten incrementan el valor de los nodos y crean distinción, se establecen nuevas relaciones, se consolidan otras ya existentes y se conforman estructuras diferenciadas por la densidad de relaciones entre componentes de las redes. De esta manera, cada red quinquenal, en su contexto, da cuenta de un evento de producción del sentido de identidad internacional de la línea de investigación.

Cada nueva publicación arbitrada, como una expresión de la impronta genética institucional, aporta elementos a la construcción del sentido de afiliación institucional al discurso dominante a nivel internacional. De acuerdo con Bourdieu, ${ }^{26}$ la construcción de este sentido conforma un capital simbólico institucional en el terreno científico-académico. Este capital, en la medida que está acreditado en revistas incluidas en índices bibliográficos, se 
interpreta como un indicador de visibilidad científica en el sistema de comunicación científica internacional. De la misma manera, los componentes de las publicaciones (autores, revistas, palabras, modelo), en la medida que se repiten, establecen relaciones, se distinguen y acumulan capital simbólico certificado en los índices bibliográficos internacionales. De acuerdo con los resultados, en el tema de la médula espinal los componentes que más se repiten, distinguen y acumulan capital son P. Rudomín, como autor; afferent, spinal, cord, cat, depolarization, and muscle, como palabras; cat, como modelo experimental; Journal of Neurophysiology, Experimental Brain Research, Journal of Physiology-London y Brain Research, como las fuentes preferidas para publicar, así como las más citadas y citantes.

\section{Conclusiones}

Cada vez que el sistema de comunicación científica internacional certifica un artículo con las marcas de P. Rudomín, spinal-cord-cat y Cinvestav-Departamento de Fisiología, ocurre una expresión de la impronta genética del Cinvestav en el contexto del discurso científico dominante del tema de la médula espinal.

Las estructuras de relaciones de comunicación resultantes entre los autores, palabras, revistas, referencias y citas permitieron mostrar la formación del capital científico-académico del Cinvestav, como un indicador de visibilidad científica construido en los índices bibliográficos internacionales.

Los términos Rudomín, spinal-cord-cat y Cinvestav se han significado, en términos de palabras claves de acceso a la literatura científica, como elementos de una misma matriz o ecuación de búsqueda de información. Conforman el código más simbólico del Cinvestav que ha mantenido la visibilidad de esta institución durante toda su historia en los índices internacionales.

Agradecimientos:

al dr. Ricardo Félix Grijalva por sus valiosos comentarios sobre los contenidos de este trabajo. Al CONACYT-Red Complejidad, Ciencias y Sociedad. 


\section{REFERENCIAS}

Albornoz, M. "La política científica y tecnológica en América Latina frente al desafío del pensamiento único”. Redes 4 (10) (1997): 95115.

Atlas de la Ciencia Mexicana 2012: Bilingual edition. México: AMC, 2 vols., 2012. Fecha de consulta: 26 de noviembre de 2014, http://atlasdelacienciamexicana.org/

Batagel, V. y A. Mrvar. Pajek. Program for Analysis and Visualization of Large Networks: Reference Manual. List of commandswith short explanation version 2.05, 2011. Fecha de consulta: 26 de noviembre de 2014, http://vlado.fmf.uni-lj.si/pub/networks/pajek/doc/pajekman. pdf

Bazerman, C. y J. Paradis. Textual Dynamics of the Professions. Historical and Contemporary Studies of Writing in Professional Communities. Wisconsin; University of Wisconsin Press, 1991.

Bazerman, C. Shaping Writing Knowledge. Wisconsin: University of Wisconsin Press, 1988.

Berkenkotter, C., T. N. Huckin y J. Ackerman, "Social Context and Social Constructed Text", en Textual Dynamics of the Professions, Charles Bazerman y James Paradis (eds.), 191-233. Wisconsin: University of Wisconsin Press, 1991.

Bourdieu, P. El oficio del científico: ciencia de la ciencia y reflexividad. Barcelona: Anagrama, 2003.

Callon, M. y B. Latour. "Unscrewing the big leviathan: How actors macrostructure reality and how sociologists help them to do so", en Advances in Social Theory and Methodology, K. D. Knorr-Cetina y A. V. Cicourel (eds.), 277-303. 1981. Boston, MA: Routledge \& Kegan Paul, 1981.

Cardinali, D. P. Neurociencia aplicada: sus fundamentos. Buenos Aires: Editorial Médica Panamericana, 2007.

Clark, B. R. The Higher Education System: Academic Organization in Cross-National Perspective. Berkeley: University of California Press, 1983.

Collazo-Reyes, F., M. E. Luna-Morales y E. Luna-Morales. "Aproximación a las formas de organización de la producción científica a través de redes de coautoría”. Avance y Perspectiva 4 (3) (2012): 1-9. Fecha de consulta: 26 de noviembre de 2014, http://avanceypers pectiva.cinvestav.mx/1866/aproximacion-a-las-formas-de-organiza cion-de-la-produccion-cientifica-a-traves-de-redes-de-coautoria

De Solla Price, D. J. Little Science, Big Science... and Beyond. Columbia: Columbia University Press, 1986.

- Little Science, Big Science. Columbia: Columbia University Press, 1963. 
Devitt, A. J. "Intertextuality in Tax Accounting; Generic, Referential and Functional", en Textual Dynamics of the Professions, Charles Bazerman y James Paradis (eds.), 336-355. Wisconsin: University of Wisconsin Press, 1991.

Diario Oficial de la Federación. Decreto de la creación del CIEA, 5 noviembre de 1960.

Doheny-Farina, S. "Creating a text / creating a Company. The role of a text in the Rise and Decline of a New Organization", en Textual Dynamics of the Professions, Charles Bazerman y James Paradis (eds.), 306-335. Wisconsin: University of Wisconsin Press, 1991.

Florida, R. y M. Kenney. "Institutions and Economic Transformation. The Case of Postwar Japanese Capitalism". Growth and Change 25 (2) (1994): 247-262.

Frigon, A. "The Cat Model of Spinal Cord Injury", en Animals Models of Spinal Cord Repair, Hakan Aldskogius (ed.), 159-186. Uppsala, Sweden: Uppsala University, Department of Neuroscience, Biomedical Center, 2013.

Garfield, E., A. I. Pudovkin y V. S. Istomin. "Why Do We Algorithmic Historiography". Journal of the American Society Information Science and Technology 54 (5) (2003): 400-412.

Guertin, P. A. "The Spinal Cord: Functional Organization, Diseases, and Dysfunctions", en Animals Models of Spinal Cord Repair, Hakan Aldskogius (ed.), 1-24. Uppsala, Sweden: Uppsala University, Department of Neuroscience, Biomedical Center, 2013.

Ibarrola, M. "La impronta genética del Cinvestav. Una mirada a la excelencia de la institución entonces y ahora", en Trayectoria de sus departamentos, secciones y unidades: 1961-2001, Cinvestav, 11-51. México: Cinvestav, 2002.

Latour, B. "On actor-network theory - A few clarifications". Soziale Welt-Zeitschrift fur Sozialwissenschaftliche Forschung und Praxis 47 (4) (1996): 369-381.

Law, J. "Notes on the theory of the actor network-ordering, strategy, and heterogeneity". Systems Practice 5 (4) (1992): 379-393.

Leydesdorff, L. "Eugene Garfield and Algorithms Historiography: Co-Words, Co-Authors and Journals". Annals of Library and Information Studies 57 (3) (2010):248-260.

Manual de Santiago. Manual de Santiago de internacionalización de la ciencia y la tecnología. Buenos Aires: REDES, 2007.

Pickstone, J. V. "A Brief Introduction to Ways of Knowing and Ways of Working”. History of Science XLIX (2011): 235-245.

"Working knowledge before and after circa 1800: Practices and disciplines in the history of science, technology and medicine". Isis 98 (3) (2007): 489-516.

Rexed, B. "A Cytoarchitectonic Atlas of the Spinal Cord in the Cat". Journal of Comparative Neurology 100 (2) (1954): 297-379.

- "The Cytoarchitectonic Organization of the Spinal Cord in the Cat". Journal of Comparative Neurology 96 (3) (1952): 415-495. 
Rosenblueth, A., J. Alanis y G. Pilar. "The Accessory Motor Innervation of the Diaphragm". Archives Internationales de Physiologie et de Biochimie 69 (1) (1961): 19-25.

Sollaci, L. B. y M. G. Pereira. "The introduction, methods, results, and discussion (IMRAD) structure: a fifty-year survey". Journal of Medical Library Association 92 (3) (2004): 364-371.

Vessuri, H. "El Centro de Investigación y de Estudios Avanzados del IPN (Cinvestav)", en 37 Modos de hacer ciencia en América Latina, B. Hernando Gómez y S. Hernán Jaramillo (comps.), 25-47. Colombia: Tercer Mundo Editores, 1997.

Para citar este artículo:

Collazo-Reyes, Francisco, María Elena Luna-Morales y Evelia Luna-Morales. 2016. "Impronta genética institucional y desarrollo de identidad internacional: estudio bibliométrico sobre médula-espinal-gato en Cinvestav." Investigación Bibliotecológica: Archivonomía, Bibliotecología e Información 70: 41-60. http://dx.doi. org/10.1016/j.ibbai.2016.10.003 